\title{
Oncological Outcomes in Japanese Men Undergoing Orchiectomy for Stage I Testicular Germ Cell Tumor
}

\author{
Ken-ichi Harada ${ }^{a}$ Hideaki Miyake $^{a}$ Takayoshi Ogawa ${ }^{b}$ Taka-aki Inoue ${ }^{c}$ Masato Fujisawa $^{a}$ \\ aDivision of Urology, Kobe University Graduate School of Medicine, Kobe; bepartment of Urology, Himeji Red Cross Hospital, Himeji; \\ 'Department of Urology, Hyogo Cancer Center, Akashi, Japan
}

\section{Key Words}

Stage I testicular germ cell tumor $\cdot$ Seminoma • Nonseminoma

\begin{abstract}
Background: The objective of this study was to retrospectively review oncological outcomes in patients with stage I testicular germ cell tumor (GCT). Patients and Methods: This study included 265 consecutive Japanese men undergoing orchiectomy for stage I testicular GCT, and a retrospective review of their records was performed. Results: Of these 265 patients, 192 and 73 were pathologically classified with seminoma and nonseminoma, respectively. Prophylactic radiation and chemotherapy were performed in 62 patients with seminoma and 6 with nonseminoma, respectively. Disease recurrence occurred in 12 seminoma patients, of whom 11 had not received prophylactic radiation therapy; however, all 12 achieved a complete response to bleomycin, etoposide and cisplatin therapy. Of the nonseminoma patients, 19 experienced disease recurrence and were then treated with bleomycin, etoposide and cisplatin followed additionally by the surgical resection of residual tumors and salvage chemotherapy in 7 and 4, respectively. There was no cancer-specific death in the 265 patients, and 5-year recurrence-free survival rates in patients with seminoma and nonseminoma were 92.6 and $72.8 \%$, respectively. Furthermore, following factors appeared to be significantly associated with recurrence-free survival in these patients: age, $T$ classification, microvascular invasion and adjuvant therapy for those with seminoma,
\end{abstract}

\section{KARGER}

Fax +4161306 1234

E-Mail karger@karger.ch

www.karger.com
(C) 2014 S. Karger AG, Basel

$1015-9770 / 14 / 0082-0084 \$ 38.00 / 0$

Accessible online at:

www.karger.com/cur and microvascular invasion for those with nonseminoma. Conclusions: Despite a generally favorable prognosis in Japanese men with stage I testicular GCT, intensive follow-up or prophylactic therapy should be considered for men with possible risk factors of disease recurrence.

Copyright $\odot 2014$ S. Karger AG, Basel

\section{Introduction}

Testicular germ cell tumor (GCT) is uncommon, but constitutes an important group of malignancies in young men. The incidence of testicular GCT has continued to increase and doubled during the last 30 years in most populations across the world; however, there is an epidemiological difference concerning this trend, which is reported to be the most marked in Northern Europe. The increases in testicular GCT remain unexplained, although changes in the prevalence of important risk factors for this disease may be responsible [1].

A diagnostic and therapeutic orchiectomy is usually performed for men with a testicular mass suspected of being testicular GCT. Approximately two thirds of all testicular GCTs are diagnosed as stage I, where the malignant lesion is confined to the testis [2]. These patients can expect to be completely cured with currently available 


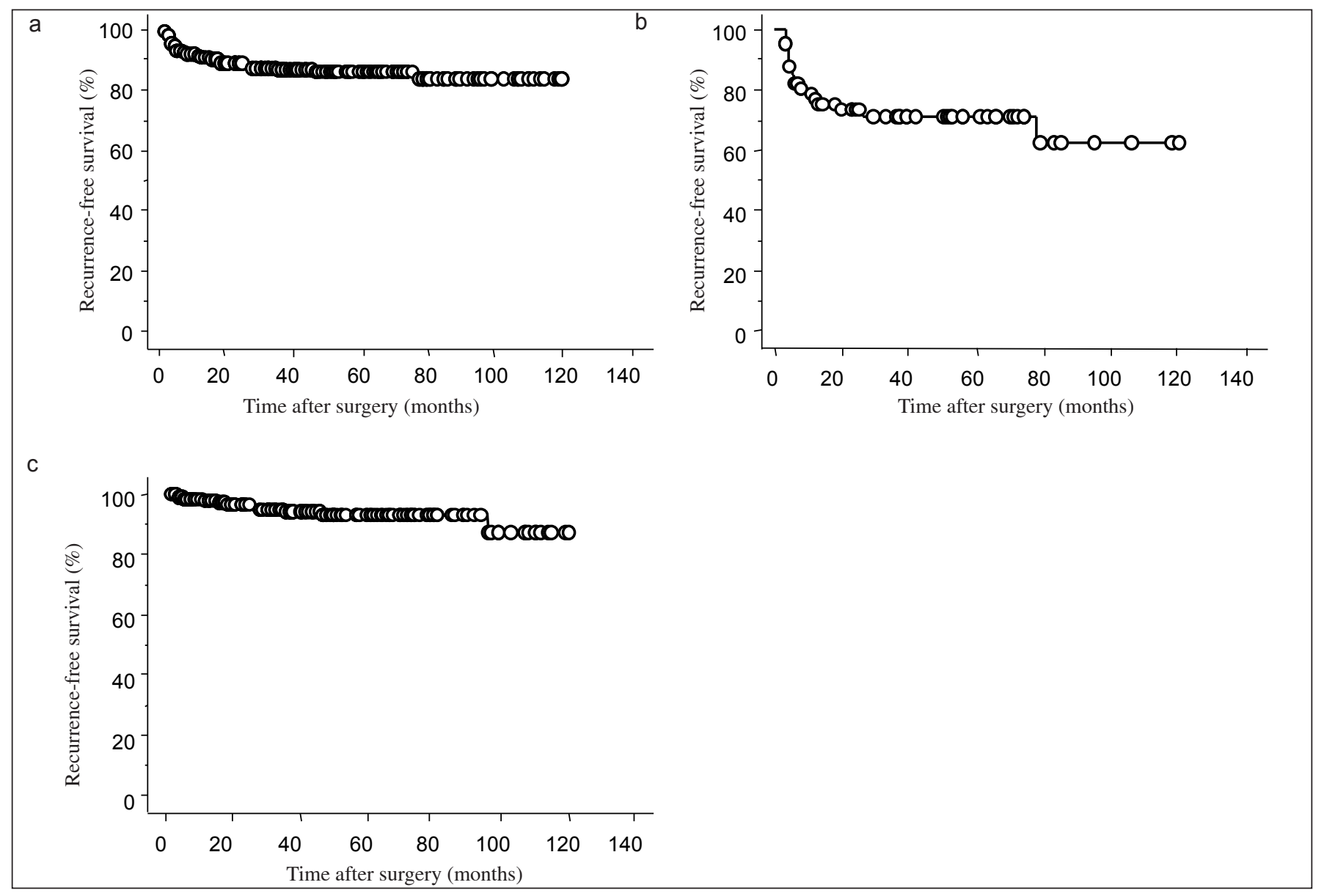

Fig. 1. a Recurrence-free survival in 265 patients with stage I testicular germ cell tumors. b Recurrence-free survival in 192 patients with stage I seminoma. c Recurrence-free survival in 73 patients with stage I nonseminoma.

therapeutic approaches, even those who develop disease recurrence; however, if treated with orchiectomy alone, the existence of undetectable micrometastases leads to the occurrence of distant recurrence in about $20 \%$ of pure seminomas and 25-30\% of nonseminomas [3, 4]. Accordingly, it is important to determine the most feasible postoperative therapeutic option based on reliable risk assessment for patients with stage I testicular GCT.

To date, there have been several studies presenting risk factors associated with disease recurrence following orchiectomy for men with stage I testicular GCT; however, most of these studies were conducted in Western countries, and the findings remain controversial [6-10]. In this study, therefore, we retrospectively reviewed the oncological outcomes in a total of 265 consecutive Japanese men undergoing orchiectomy for stage I testicular GCT at our institutions in order to identify factors predicting postoperative disease recurrence in this cohort of patients.

\section{Patients and Methods}

This study included a total of 265 consecutive Japanese men who underwent radical inguinal orchiectomy for stage I testicular GCT at our institutions between January 2001 and December 2010. Informed consent to perform this study was obtained from all patients, and the study design was approved by the Research Ethics Committee of our institutions.

The staging procedures at our institutions included physical examinations, brain, thoracic, abdominal and/or pelvic computed tomography, bone scan and the measurement of tumor markers, including $\alpha$-fetoprotein, human chorionic gonadotropin $\beta$ subunit (HCG- $\beta$ ) and lactate dehydrogenase. All pathological specimens were examined under the guidance of a single experienced genitourinary pathologist.

Following the diagnosis of the histological subtype, all patients were thoroughly informed about their estimated risk of disease recurrence and postoperative treatment options, including active surveillance, radiotherapy, chemotherapy and retroperitoneal lymph node dissection. At our institutions, radiotherapy has been recommended if patients with stage I seminoma wish to receive prophylactic adjuvant therapy, while active surveillance has been 


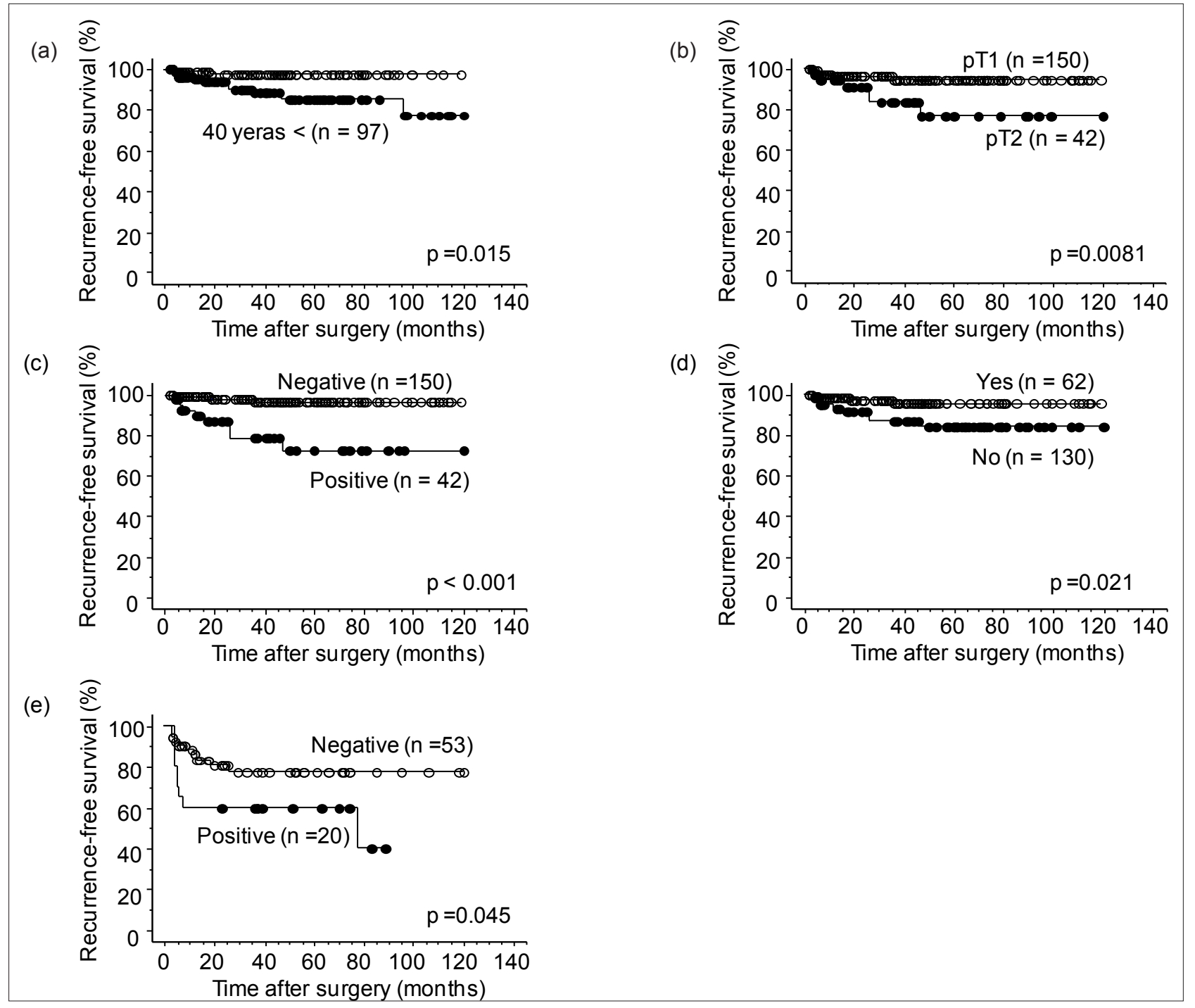

Fig. 2. Recurrence-free survival in patients with stage I seminoma according to the (a) age, (b) T classification, (c) microvascular invasion and (d) adjuvant therapy and in those with stage I seminoma according to the (e) microvascular invasion.

the preferred postoperative therapeutic option for stage I nonseminoma patients. The principle postoperative follow-up schedule was as follows: measurement of tumor markers and radiological examinations every 3 months for 1 year after orchiectomy, and then every 6 months until 5 years after surgery.

In this study, all statistical examinations were performed using the Statview 5.0 software (Abacus Concepts, Inc., Berkeley, CA, USA), and $\mathrm{p}$ values $<0.05$ were considered significant. A chi-squared test was used to analyze the association between several factors and disease recurrence according to each histological subtype. Recurrence-free survival was defined as the time from orchiectomy to the detection of disease recurrence or was censored at the date of last follow-up, and based on this definition, the recurrence-free survival rates were calculated with the KaplanMeier method, and differences were determined by the log-rank test.

\section{Results}

Of the 265 patients included in this study, 192 $(72.5 \%)$ and $73(27.5 \%)$ were pathologically diagnosed with seminoma and nonseminoma, respectively. Of the 192 patients with seminoma, $62(32.3 \%)$ received postoperative prophylactic radiotherapy, while prophylactic chemotherapy was performed in $6(8.2 \%)$ of the 73 with nonseminoma. During the observation period of this study (median, 34 months), all patients survived; however, disease recurrence occurred in 31 patients, and the 1-, 3-, 5- and 10-year recurrence-free survival rates were 91.6, 87.2, 86.3 and $81.5 \%$, respectively (fig. 1A). 
Table 1. Patient characteristics according to disease recurrence

\begin{tabular}{|c|c|c|c|c|c|c|}
\hline & \multicolumn{3}{|c|}{ Seminoma $(n=192)$} & \multicolumn{3}{|c|}{ Nonseminoma $(\mathrm{n}=73)$} \\
\hline & \multicolumn{2}{|c|}{ Disease recurrence } & \multirow[b]{2}{*}{$\mathrm{p}$} & \multicolumn{2}{|c|}{ Disease recurrence } & \multirow[b]{2}{*}{$\mathrm{p}$} \\
\hline & $\begin{array}{l}\text { Yes } \\
(\mathrm{n}=12)\end{array}$ & $\begin{array}{l}\text { No } \\
(\mathrm{n}=180)\end{array}$ & & $\begin{array}{l}\text { Yes } \\
(\mathrm{n}=12)\end{array}$ & $\begin{array}{l}\text { No } \\
(\mathrm{n}=180)\end{array}$ & \\
\hline Age (years) & $34.0 \pm 5.5$ & $42.4 \pm 11.1$ & 0.015 & $38.1 \pm 13.8$ & $34.2 \pm 12.8$ & 0.10 \\
\hline Tumor size $(\mathrm{cm})$ & & & 0.19 & & & 0.26 \\
\hline$\leq 4.0$ & 2 & 63 & & 5 & 22 & \\
\hline$>4.0$ & 10 & 117 & & 14 & 32 & \\
\hline $\mathrm{T}$ dassification & & & 0.012 & & & 0.17 \\
\hline T 1 & 6 & 145 & & 13 & 45 & \\
\hline T 2 & 6 & 35 & & 6 & 9 & \\
\hline Serum AFP & & & - & & & 0.57 \\
\hline Normal & - & - & & 4 & 15 & \\
\hline Abnormal & - & - & & 15 & 39 & \\
\hline Serum HCG- $\beta$ & & & 0.24 & & & 0.074 \\
\hline Normal & 7 & 83 & & 5 & 27 & \\
\hline Abnormal & 5 & 97 & & 14 & 27 & \\
\hline Microvascular invasion & & & $<0.001$ & & & 0.028 \\
\hline Negative & 5 & 143 & & 10 & 43 & \\
\hline Positive & 7 & 34 & & 9 & 11 & \\
\hline Adjuvant therapy & & & 0.0016 & & & 0.057 \\
\hline Yes & 11 & 119 & & 19 & 45 & \\
\hline No & 1 & 61 & & 0 & 9 & \\
\hline
\end{tabular}

$\mathrm{AFP}=\alpha$-Fetoprotein $;$ HCG- $\beta=$ human chorionic gonadotropin $\beta$.

Of the 31 patients with disease recurrence, 12 (38.7\%) and $19(61.3 \%)$ were classified with seminoma and nonseminoma, respectively. Of the 68 patients who received prophylactic treatment, only 1 patient $(1.5 \%)$ with seminoma developed disease recurrence after radiation therapy. Following disease recurrence, all 12 seminoma patients achieved a complete response to BEP (bleomycin, etoposide and cisplatin) therapy, while 19 nonseminoma patients were initially treated with BEP followed by additional treatments, including surgical resection of residual tumor masses and salvage chemotherapy in 7 and 4, respectively. Pathological examinations of resected tumor tissues in these 7 nonseminoma patients revealed either no viable cancer cells or mature teratoma. As shown in figure 1B and C, the 1-, 3-, 5- and 10-year recurrence-free survival rates in seminoma patients were 97.7, 93.8, 92.6 and $91.5 \%$, respectively, while those in nonseminoma patients were $78.4,72.8,72.8$ and $64.7 \%$, respectively.

Table 1 shows the association between several parameters and disease recurrence after orchiectomy. In patients with seminoma, despite the lack of a significant impact of the tumor size, pretreatment serum HCG- $\beta$ value and adjuvant therapy, disease recurrence was sig- nificantly correlated with the age, $T$ classification and microvascular invasion. In patients with nonseminoma, disease recurrence was significantly affected by the microvascular invasion, but not the tumor size, $T$ classification, pretreatment serum HCG- $\beta$ and $\alpha$-fetoprotein values and adjuvant therapy. We subsequently analyzed the recurrence-free survival according to several risk factors in patients with stage I testicular GCT. As shown in table 2 , following factors appeared to be significantly associated with recurrence-free survival in these patients: age, $\mathrm{T}$ classification, microvascular invasion and adjuvant therapy for those with seminoma, and microvascular invasion for those with nonseminoma. Recurrence-free survival curves according to significant risk factors are presented in figure 2 .

\section{Discussion}

It has been well documented that, if primary diseases are diagnosed with stage I testicular GCT, orchiectomy alone cures $80 \%$ of seminoma and $70-75 \%$ of nonseminoma [3, 4]; however, recurrent diseases, irrespective of their histological subtypes, can be successfully treated, 
Table 2. Recurrence-free survival according to various parameters

\begin{tabular}{|c|c|c|c|c|}
\hline & \multicolumn{2}{|l|}{ Seminoma $(n=192)$} & \multicolumn{2}{|c|}{ Nonseminoma $(\mathrm{n}=73)$} \\
\hline$\leq 40$ & 85.9 & & 79.0 & \\
\hline$>40$ & 97.6 & & 50.1 & \\
\hline Tumor size $(\mathrm{cm})$ & & 0.18 & & 0.24 \\
\hline $\mathrm{T}$ classification & & 0.0079 & & 0.16 \\
\hline $\mathrm{T} 1$ & 90.4 & & 76.9 & \\
\hline $\mathrm{T} 2$ & 96.4 & & 57.1 & \\
\hline Serum AFP & & - & & 0.50 \\
\hline Normal & - & & 77.8 & \\
\hline Abnormal & - & & 70.5 & \\
\hline Serum HCG- $\beta$ & & 0.96 & & 0.11 \\
\hline Positive & 74.9 & & 57.9 & \\
\hline Adjuvant therapy & & 0.0016 & & - \\
\hline Yes & 88.8 & & 68.5 & \\
\hline No & 98.0 & & 100 & \\
\hline
\end{tabular}

$\mathrm{AFP}=\alpha$-fetoprotein HCG- $\beta=$ human chorionic gonadotropin $\beta$.

resulting in the achievement of an overall long-term cure rate of $98-99 \%$ in this category of patients [2]. Accordingly, considering the risks and benefits of prophylactic therapeutic modalities, the optimal treatment following orchiectomy for stage I disease remains controversial in patients with both seminoma and nonseminoma; that is, the disadvantage of surveillance is the certain proportion of patients who will be later treated with salvage chemotherapy, known to cause long-term toxicities, whereas a routine prophylactic treatment strategy for stage I testicular GCT as an adjuvant setting results in the exposure of a high proportion of patients to overtreatment accompanying several adverse events [11]. Furthermore, despite the presence of epidemiological differences in the characteristics of testicular GCT [1], the majority of studies on this disease were based on data from Western populations. Taken together, we retrospectively reviewed the oncological outcomes in 265 consecutive Japanese patients with stage I testicular GCT who underwent high orchiectomy at our institutions in order to characterize the clinical course and identify risk factors associated with disease recurrence in this cohort of patients.
At our institutions, based on the concept that the reduced risk of postoperative recurrence from the use of adjuvant therapies should be balanced against the potential adverse events in patients with stage I testicular GCT, it has been decided whether or not adjuvant radiation therapy should be performed for stage I seminoma according to the preference of each patient, whereas prophylactic adjuvant chemotherapy has not usually been recommended for stage I nonseminoma due to severe side effects of cisplatin-based combination chemotherapy. As a result, in this series, $62(32.3 \%)$ of the 192 patients with stage I seminoma received adjuvant radiotherapy, while adjuvant chemotherapy was performed in only $6(8.2 \%)$ of the 73 patients with stage I nonseminoma. Of these 68 who received adjuvant therapy, only 1 (1.5\%) seminoma patient who received prophylactic radiotherapy developed disease recurrence, suggesting the utility of adjuvant therapy for stage I testicular GCT. In fact, there have been several studies advocating adjuvant treatment in patients with stage I testicular GCT, particularly those with nonseminoma based on risk-adopted policies [12, 13]; however, it is also documented that at least a third of 
patients even classified into the so-called high-risk group are unnecessarily treated with a risk-adopted policy [14, $15]$.

In this series, the 5-year recurrence-free survival rates in patients with seminoma and nonseminoma were 92.6 and $72.8 \%$, respectively, and there was no cancer-specific death. Although it is difficult to compare the present oncological outcomes with those in other reports due to various differences in the characteristics and therapeutic policies among these studies, the prognosis of patients with stage I testicular GCT treated at our institutions seems to be comparable to those in previous studies [6, 10, 12-16]. For example, Sturgeon et al. [16] evaluated the prognostic outcomes in patients with stage I nonseminomatous GCT who initially underwent active surveillance with delayed treatment at relapse, and reported that the estimated 5-year recurrence-free and disease-specific survival rates were 72.5 and $99.3 \%$, respectively. These findings suggest that it might be possible to achieve oncological outcomes in Japanese men with stage I testicular GCT similar to those in Western populations based on the strategy concerning postoperative management adopted at our institutions.

Despite the generally favorable oncological outcomes in patients with stage I testicular GCT, it is important to identify risk factors closely associated with disease recurrence following orchiectomy in order to further reduce the recurrence risk by providing prophylactic therapy as an adjuvant setting. To date, there have been several risk factors identified for disease recurrence following orchiectomy for stage I testicular GCT; however, the outcomes have been controversial [6-11, 17]. For example, Warde et al. [17] analyzed the 4 largest studies of surveillance in stage I seminoma including 638 cases and found the independent impact of the tumor size and invasion of the rete testis on postoperative disease recurrence; however, a recent attempt to prospectively validate these findings failed [6]. In addition, the presence of an embryonal cell carcinoma element has been shown to be a significant risk factor for relapse in stage I nonseminoma patients in several studies, but its importance in multivariate models is not consistent [11]. In this series, of several factors examined, age, T classification, microvascular invasion and adjuvant therapy were significantly associated with recurrence-free survival in patients with seminoma, while only microvascular invasion had a significant impact on recurrence-free survival in those with nonseminoma. Collectively, these findings suggest that it is necessary to perform a prospective study including a much larger number of both patients with seminoma and those with nonseminoma in order to develop a system based on reliable risk factors that enables a more precise prediction of postoperative disease recurrence for patients with stage I testicular GCT.

Here, we would like to emphasize several limitations of this study. Firstly, this was a retrospective study including a relatively small number of patients, and postoperative managements for these patients were not conducted based on strictly regulated criteria. Secondly, progress in radiological examinations as well as therapeutic modalities during the observation period may have affected the outcomes of this study. Thirdly, it was impossible to obtain detailed pathological findings from all patients included in this study; therefore, the prognostic significance of important pathological factors, such as rete testis invasion in seminoma and percent of embryonal carcinoma element in nonseminoma, couls not be assessed. Finally, although this study focused on oncological outcomes alone, it is important to more comprehensively assess the clinical outcomes, such as adverse events and quality of life, considering the younger age of this category of patients.

In conclusion, we retrospectively reviewed the data from 265 Japanese men with stage I testicular GCT who underwent high orchiectomy, and found that their oncological outcomes were generally favorable. However, despite the absence of patients who died during the observation period of this study, 31 patients $(11.7 \%)$ developed disease recurrence, and based on these findings, this study identified several risk factors significantly associated with recurrence-free survival, including age, $\mathrm{T}$ classification, microvascular invasion and adjuvant therapy for those with seminoma, and microvascular invasion for those with nonseminoma. Collectively, these findings indicate that intensive follow-up or prophylactic therapy should be considered for men with such possible risk factors of disease recurrence. 


\section{References}

-1 Purdue MP, Devesa SS, Sigurdson AJ, McGlynn KA: International patterns and trends in testis cancer incidence. Int J Cancer 2005; 115:822-827.

- 2 Gori S, Porrozzi S, Roila F, Gatta G, De Giorgi U, Marangolo M: Germ cell tumours of the testis. Crit Rev Oncol Hematol 2005; 53:141-164.

- 3 Warde P, Huddart R, Bolton D, Heidenreich A, Gilligan T, Fossa S: Management of localized seminoma, stage I-II: SIU/ICUD Consensus Meeting on Germ Cell Tumors (GCT), Shanghai 2009. Urology 2011; 78:S435-S443.

-4 Stephenson AJ, Aprikian AG, Gilligan TD, Oldenburg J, Powles T, Toner GC, Waters WB: Management of low-stage nonseminomatous germ cell tumors of testis: SIU/ ICUD Consensus Meeting on Germ Cell Tumors (GCT), Shanghai 2009. Urology 2011; 78:S444-S455.

$\checkmark 5$ Chung P, Mayhew LA, Warde P, Winquist E, Lukka H: Management of stage I seminomatous testicular cancer: a systematic review. Clin Oncol 2010;22:6-16.

6 Cullen M: Surveillance or adjuvant treatments in stage 1 testis germ-cell tumours. Ann Oncol 2012;23 (Suppl 10):342-348.

7 Tan A, Gilligan T: Controversies in the management of early-stage germ cell tumors. Curr Oncol Rep 2009;11:235-243. $\checkmark 8$ Choueiri TK, Stephenson AJ, Gilligan T, Klein EA: Management of clinical stage I nonseminomatous germ cell testicular cancer. Urol Clin North Am 2007;34:137-148.

-9 Hotte SJ, Mayhew LA, Jewett M, Chin J, Winquist E: Management of stage I non-seminomatous testicular cancer: a systematic review and meta-analysis. Clin Oncol 2010; 22:17-26.

10 Kollmannsberger C, Moore C, Chi KN, Murray N, Daneshmand S, Gleave M, Hayes-Lattin B, Nichols CR: Non-risk-adapted surveillance for patients with stage I nonseminomatous testicular germ-cell tumors: diminishing treatment-related morbidity while maintaining efficacy. Ann Oncol 2010;21: 1296-1301.

11 de Wit R, Fizazi K. Controversies in the management of clinical stage I testis cancer. J Clin Oncol 2006;24:5482-5492.

12 Maroto P, García del Muro X, Aparicio J, Paz-Ares L, Arranz JA, Guma J, Terrassa J, Barnadas J, Dorta J, Germà-Lluch JR: Multicentre risk-adapted management for stage I non-seminomatous germ cell tumours. Ann Oncol 2005;16:1915-1920.

-13 Amato RJ, Ro JY, Ayala AG, Swanson DA: Risk-adapted treatment for patients with clinical stage I nonseminomatous germ cell tumor of the testis. Urology 2004;63:144-148.
4 Tandstad T, Dahl O, Cohn-Cedermark G, Cavallin-Stahl E, Stierner U, Solberg A, Langberg C, Bremnes RM, Laurell A, Wijkstrøm $\mathrm{H}$, Klepp O: Risk-adapted treatment in clinical stage I nonseminomatous germ cell testicular cancer: the SWENOTECA management program. J Clin Oncol 2009;27:2122-2128.

15 Albers P, Siener R, Kliesch S, Weissbach L, Krege S, Sparwasser C, Schulze H, Heidenreich A, de Riese W, Loy V, Bierhoff E, Wittekind C, Fimmers R, Hartmann M: Risk factors for relapse in clinical stage I nonseminomatous testicular germ cell tumors: results of the German Testicular Cancer Study Group Trial. J Clin Oncol 2003;21:1505-1512.

16 Sturgeon JF, Moore MJ, Kakiashvili DM, Duran I, Anson-Cartwright LC, Berthold DR, Warde PR, Gospodarowicz MK, Alison RE, Liu J, Ma C, Pond GR, Jewett MA: Non-riskadapted surveillance in clinical stage I nonseminomatous germ cell tumors: the Princess Margaret Hospital's experience. Eur Urol 2011;59:556-562.

17 Warde P, Specht L, Horwich A, Oliver T, Panzarella T, Gospodarowicz M, von der Maase H. Prognostic factors for relapse in stage I seminoma managed by surveillance: a pooled analysis. J Clin Oncol 2002;20:4448-4452. 